\title{
Napping and Less Disturbed Nighttime Sleep Associated with Reduced Carotid Intima-Media Thickness in Elderly Alzheimer Caregivers
}

\author{
Jennifer Schwartz ${ }^{\mathrm{a},}$, Matthew A. Allison ${ }^{\mathrm{b}}$, Sonia Ancoli-Israel ${ }^{\mathrm{c}, \mathrm{e}}$, Melbourne F. Hovell ${ }^{\mathrm{d}}$, Ruth E. \\ Patterson $^{\mathrm{b}, \mathrm{e}}$, Loki Natarajan ${ }^{\mathrm{b}, \mathrm{e}}$, Simon J. Marshall ${ }^{\mathrm{f}}$ and Igor Grant ${ }^{\mathrm{c}}$
}

\author{
${ }^{a}$ San Diego State University/University of California, San Diego, Joint Doctoral Program in Public Health, San Diego, CA \\ 5500 Campanile Drive, San Diego, CA 92182, USA \\ ${ }^{b}$ Department of Family and Preventive Medicine, University of California, San Diego, La Jolla, CA, USA \\ ${ }^{c}$ Department of Psychiatry, University of California, San Diego, La Jolla, CA, USA \\ ${ }^{d}$ Center for Behavioral Epidemiology and Community Health and Division of Health Promotion and Behavioral \\ Sciences, Graduate School of Public Health, San Diego State University, San Diego, CA, USA \\ ${ }^{e}$ Moores UCSD Cancer Center, University of California, San Diego, La Jolla, CA, USA \\ ${ }^{f}$ School of Exercise and Nutritional Sciences, San Diego State University, San Diego, CA, USA
}

\begin{abstract}
This study tested the hypothesis that disturbed or very short or long sleep durations are associated with subclinical atherosclerosis, as measured by carotid intima-media thickness (CIMT), in a sample of community-dwelling elderly spousal caregivers. Participants were 126 spousal caregivers of a person with Alzheimer's disease who underwent in-home sleep assessment by wrist actigraphy for 72 consecutive hours. Carotid artery ultrasound, also conducted in participants' homes, was used to measure CIMT in millimeters. Linear regression models including covariates related to sleep and CVD indicated that a longer daytime sleep duration was associated with significantly reduced mean common CIMT $(B=-0.04, p=0.02)$. Specifically, for every additional hour of sleep obtained during the day, mean common CIMT was reduced by $0.04 \mathrm{~mm}$. Conversely, greater night time wake after sleep onset (WASO) was associated with significantly increased mean common CIMT $(\mathrm{B}=0.04, \mathrm{p}=0.05)$; for each additional hour of WASO, mean common CIMT increased by $0.04 \mathrm{~mm}$. When combinations of sleep parameters were examined, caregivers who slept $<30$ minutes during the day and had $\geq 1$ hour of WASO throughout the night had significantly greater $(B=0.06, p=0.04)$ mean common CIMT than caregivers who slept $<30$ minutes during the day and had $<1$ hour of WASO. These analyses suggest that shorter naps and more disturbed nighttime sleep are associated with increased CIMT. Interventions that enhance sleep quantity and reduce sleep disturbance may decrease the prevalence and extent of subclinical atherosclerosis in older caregivers. Longitudinal studies with objective sleep measures that examine the effects of napping and disturbed sleep on subclinical atherosclerosis are needed.
\end{abstract}

Keywords: Napping, sleep disturbance, subclinical atherosclerosis, carotid intima-media thickness, carotid artery ultrasound, caregivers.

\section{INTRODUCTION}

Spousal caregivers of people with Alzheimer's disease may experience frequent awakenings during sleep due to challenges associated with caregiving [1]. This can lead to a state of prolonged and heightened sympathetic arousal [2], which in turn is thought to promote pathologic processes related to the initiation and progression of atherosclerotic cardiovascular disease (CVD) [3, 4]. Indeed, caregivers have been found to experience more disrupted sleep, with about two-thirds of the ten million spousal caregivers of people with Alzheimer's disease in the United States reporting

*Address correspondence to this author at the San Diego State University/University of California, San Diego, Joint Doctoral Program in Public Health, San Diego, CA, USA; Tel: (619)787-9668;

E-mail: jeschwartz@ucsd.edu routine sleep disturbance [5]. At the same time, this group is at increased risk for CVD and death compared to noncaregivers [1]. The increased risk of CVD could, in part, be due to the effect that insufficient sleep has on numerous physiologic processes that can hasten atherosclerotic progression and risk of CVD [6].

Although the underlying mechanisms of the adverse effects of insufficient sleep on atherosclerosis are not completely understood, numerous pathways have been proposed. Researchers hypothesize that insufficient sleep may cause sympathoadrenal medullary arousal and elevated inflammatory and coagulation markers, leading to increased coronary artery calcification as well as increased risk for CVD events [7]. For example, Werle et al. found that selfreported total hours of sleep (including both nighttime sleep and naps) were inversely related to the 9-year risk of cardiovascular death among a cohort of 187 participants over 
the age of 80 years [8]. A systematic review of 15 prospective studies by Cappuccio et al. concluded that short ( $\leq 5-6$ hours) and long ( $>8-9$ hours) sleep durations were significantly associated with increased risk of coronary heart disease [9]. Importantly, insufficient sleep has been associated with increases in obesity, diabetes, and hypertension, all of which are strong predictors of future development of CVD [10]. However, the ability to accurately predict risk of CVD based on these traditional risk factors (obesity, diabetes, and hypertension) appears to weaken with increasing age [11]. Therefore, research to identify novel risk factors (e.g. sleep quantity and quality) associated with cardiovascular outcomes is especially important in the elderly.

Atherosclerosis in the carotid arteries is a marker of generalized atherosclerosis [12]. Carotid intima-media thickness (CIMT) - the distance between the interfaces of the lumen-intima and the media-adventitia boundaries - is a surrogate marker of atherosclerosis [13, 14]. Larger CIMT is due to the accumulation of lipid, smooth muscle cells and inflammatory infiltrate that are part of the atherosclerotic process [12]. In this regard, a greater CIMT is associated with CVD risk factors such as older age, higher body mass index (BMI) and cholesterol levels, male gender and the presence of diabetes, hypertension, and smoking. Moreover, CIMT has been shown to be a strong predictor of future CVD and events $[13,14]$.

Literature on relationships of sleep duration and quality with subclinical carotid atherosclerosis is limited. In the first of only three known studies that investigated the relationship between sleep and CIMT, Wolff et al. reported that both short (5 hours) and long (11 to 12 hours) self-reported total (night and day) sleep durations were associated with greater CIMT in a large $(n=2,437)$ cross-sectional analysis [15]. The second study reported no association between self-reported insomnia (defined as difficulty falling asleep, maintaining sleep, and early morning awakening) and CIMT in a population-based cohort study of 1,605 participants with a mean age of 65 years [16]. The third and most recent crosssectional analysis on 2,214 men and women over the age of 40 years indicated that long self-reported sleep ( $\geq 7$ hours) was associated with a greater risk of CIMT $\geq 1.2 \mathrm{~mm}$ compared with a sleep duration of 6 hours (OR, 1.26; 95\%CI, 1.03-1.54) [17].

While evidence supporting a link between insufficient sleep and arterial disease is accumulating, data on objectively measured sleep and cardiovascular risk factors in older adults are limited and conflicting [18]. Research examining these associations in elderly dementia caregivers is even more limited, yet this group generally experiences frequent sleep difficulties, thereby providing greater opportunity for sleep to impact health [19]. Therefore, the purpose of this cross-sectional analysis was to examine whether objectively measured sleep quantity and disturbed sleep were associated with atherosclerosis in the carotid arteries from ultrasound imaging among 126 communitydwelling elderly spousal caregivers of a person with Alzheimer's disease who participated in the Alzheimer Caregiver Coping Study. It was hypothesized that chronically inadequate sleep duration and disturbed sleep increase the possibility for arterial damage and downstream CVD.

\section{METHODS}

\section{The Alzheimer Caregiver Coping Study}

The Alzheimer Caregiver Coping Study was conducted at the University of California, San Diego (UCSD) to examine the relationships between physiological and psychological stress markers, and health risk factors in spousal caregivers of a person with Alzheimer's disease. All data were collected between 2007 and 2010.

Participants were 126 community-dwelling men and women over the age of 55 years who were married, living with, and providing continuous in-home care to a spouse diagnosed with Alzheimer's disease. Caregivers were recruited via referrals from the UCSD Alzheimer's Disease Research Center, community Alzheimer caregiver support groups, local agencies serving caregivers, recommendations from other participants enrolled in the study, flyers, media advertisements, and senior health fairs. Participants provided written informed consent, and the study was approved by the UCSD Institutional Review Board.

Caregivers were excluded if they had a current diagnosis or treatment for a life-threatening or terminal medical condition that required ongoing care (i.e. advanced CVD, Parkinson's disease, and/or a severe psychiatric disorder), extreme hypertension $(>200 / 120 \mathrm{~mm} \mathrm{Hg})$, current or recent (within the past 5 years) treatment for cancer, organ transplantation requiring anti-rejection medication, or use of corticosteroids, $\beta$-blocking, and/or anticoagulant medication.

\section{Measures}

All measurements and assessments, other than objective measures of sleep, were administered between 9:00AM and 11:00AM in participants' homes by trained research personnel, which included a nurse, research assistant, and certified sonographer. The research assistant administered a semi-structured interview that gathered information on sociodemographics, years of caregiving, psychosocial functioning, caregiving overload, health behaviors, medical history, hospitalizations, and alcohol/drug history. Fasting venous blood was drawn within one week of the initial visit between 9:00AM and 11:00AM.

\section{Sociodemographics, Medical Data, and Past Health History}

Sociodemographic information such as age, gender, education, and occupation were collected by interview. History of cardiovascular disease was defined as previous heart attack, heart failure, angina, heart disease, stroke, or transient ischemic attack. Caregivers were asked if they had ever been told by a physician that they had type 2 diabetes, hypertension, dyslipidemia, myocardial infarction or stroke, with answers coded yes/no. Information about cardiovascular medication (i.e., aspirin, angiotensin-converting enzyme inhibitors, and statins) and antidepressant use (i.e., atypicals, selective serotonin reuptake inhibitors, tricyclics, etc.) was obtained via participant report and confirmed by examination of the medication containers. Smoking status, alcohol consumption, and physical activity information was also collected during the home visit.

Smoking status was categorized as current/former smoker vs. never smoker. Alcohol consumption was 
quantified in terms of the number of alcoholic drinks consumed per week. Data on height and weight were obtained by participant report, and body mass index (BMI) was calculated as the ratio of self-reported weight in kilograms to height in meters squared. Participants with a BMI $>30$ were classified as obese. Blood pressure was measured three times using a non-invasive Microlife BP monitor (model number 3AC1-1PC) with the caregiver in a supine position. The average of the three measurements was used to create a composite resting blood pressure estimate. Hypertension was defined either by self-report of physiciandiagnosed hypertension and current use of a prescription antihypertensive, or as a resting diastolic blood pressure or systolic blood pressure $\geq 90$ or $\geq 140 \mathrm{mmHg}$, respectively. Dyslipidemia was defined either by total-to-HDL ratio $>5$, or by self-report of current use of prescription cholesterollowering medications. Diabetes was defined either by selfreport of physician-diagnosed diabetes and current use of antiglycemic medication, or a fasting blood glucose $\geq 126 \mathrm{mg} / \mathrm{dL}$, as classified by the American Diabetes Association [20].

\section{Psychological Questionnaires}

The semi-structured interview included questions that evaluated duration of caregiving, psychological distress, perceived stress, depressive symptom severity, and anxiety and depressive symptoms. The 10-item short form of the Center for Epidemiologic Studies Depression Scale (CESD10) was used to assess depressive symptoms [21]. Levels of life stress were assessed with the 4-item Role Overload Scale, which measured the extent to which participants felt overwhelmed by everyday tasks. Reliability (Cronbach's alpha) for the CESD-10 and the 4-item Role Overload Scale in our sample of caregivers was 0.52 and 0.82 , respectively. Lower role overload and CESD-10 scores indicate lower stress and depressive symptoms, respectively.

\section{Objective Sleep Measure}

Objective sleep/wake activity was measured with the Actiwatch-Light ${ }^{\circledR}$ (Mini Mitter Co., Inc, a Respironics, Inc. Co., Bend, OR), which was worn for 72 consecutive hours on participants' non-dominant wrist. Actigraphy has been validated in and recommended for use among elderly populations and has been compared favorably with polysomnography (PSG), which is deemed the gold standard for sleep assessment [22]. The Actiwatch-Light ${ }^{\circledR}$ uses a piezoelectric linear accelerometer (sensitivity $<0.01$ g-force) with a sampling rate of $32 \mathrm{~Hz}$ to measure and record wrist movement. Movement, which was measured as the number of accelerations per minute, was captured via internal motion sensors in the watch. Calculating wrist activity over time allowed for an objective measure of duration and disruption of sleep. The recorded actigraphy data were analyzed using Actiware $^{\circledR}$ sleep and activity monitoring software (version 5, by Mini Mitter/Respironics/Philips). A high sensitivity setting and a minimum minor rest interval of 10 minutes were adopted to score sleep with the software. Sleep diaries completed by participants estimated times the actigraphy watch was removed, bedtimes, and wake times, and were used for editing the actigraphy data.

The following sleep parameters used in the present analysis were averaged across the three consecutive 24-hour time periods and selected a priori: 1) total nighttime sleep duration (hours per night spent sleeping from reported bedtime to final uptime); 2) total hours of daytime sleep (nap time), with naps defined as no activity for a minimum of 10 minutes; and 3) hours awake after sleep onset (WASO) between reported bedtime and final uptime, which was used to denote sleep disturbance.

\section{Outcomes - Ultrasound Imaging of Carotid Artery Intima-Media Thickness}

Carotid artery ultrasound was conducted with the participant lying in the supine position, using an Acuson Cypress Portable Ultrasound Unit with a 5.4-6.6 MHz Acuson 7L3 transducer. High resolution B-mode ultrasound images were taken of the near and far walls of the common carotid artery section from two uniform interrogation angles per vessel (left: $180^{\circ}$ and $240^{\circ}$; right: $120^{\circ}$ and $180^{\circ}$ ). Segments were defined by the carotid flow divider, which was used as a reference point: the common carotid was defined as the segment 1 to $2 \mathrm{~cm}$ proximal to the flow divider. Imaging of all participants was conducted by the same certified sonographer to avoid inter-sonographer inconsistency.

The computer program Vascular Research Tools (Medical Imaging Applications, Coralville, IA) was used to analyze carotid intima-media thickness (CIMT) by a blinded image reader. Carotid intima-media thickness $(\mathrm{mm})$ was defined as the distance between the lumen-intima boundary and the media-adventitia boundary. The mean of the means measured from the far walls of the common carotid artery segment, which will be referred to as the "common CIMT", was examined in the current study.

\section{Statistical Analysis}

Associations between continuous sleep measures, key covariates (e.g. hypertension), and CIMT were tested using Pearson $r$ and t-tests, and potential confounders were identified. First, the sleep variables were treated as continuous. In secondary analyses, to test for non-linear associations or thresholds, sleep parameters were modeled categorically by combining sleep parameters into three new variables: 1) nighttime sleep duration with daytime sleep duration; 2) nighttime sleep duration with WASO; and 3) daytime sleep duration with WASO. In these secondary analyses, reference groups and cut points were chosen based on current viewpoints about clinically significant cut points of these sleep parameters in elderly adults, and daytime sleep duration was reported in minutes for ease of interpretation. Specifically, 7-8 hours of sleep at night, $<1$ hour of WASO throughout the night, and $<30$ minutes of daytime sleep are generally considered the normal cut-offs for elderly adults, and were therefore used as the reference groups and/or cut points [23].

Multivariable linear regression analysis was employed to identify which sleep parameters were significantly associated with CIMT independent of covariates. In the multiple regression models, covariates were restricted to age, gender, those variables showing significant $(\mathrm{p}<0.10)$ univariate correlations with the exposure variables (sleep parameters) or the outcome variable (CIMT), and variables selected apriori likely to be related to atherosclerosis and sleep. Covariates adjusted for in Model 1 included age and gender. 
Table 1. Characteristics of a Sample of Elderly Spousal Caregivers of a Person with Alzheimer's Disease $(\mathrm{N}=126)$ in a Study of the Associations of Sleep with Subclinical Atherosclerosis

\begin{tabular}{|c|c|}
\hline Age (years), mean (SD) & $74.16(7.98)$ \\
\hline Female, n (\%) & $89(71.20)$ \\
\hline \multicolumn{2}{|l|}{ Ethnicity, n (\%) } \\
\hline Caucasian & $115(92.00)$ \\
\hline Non-Caucasian & $10(8.00)$ \\
\hline Education (years), mean (SD) & $15.15(3.05)$ \\
\hline Body mass index $\left(\mathrm{kg} / \mathrm{m}^{2}\right)$, mean $(\mathrm{SD})$ & $26.49(4.71)$ \\
\hline Systolic blood pressure (mmHg), mean (SD) & $134.3(15.3)$ \\
\hline Diastolic blood pressure (mmHg), mean (SD) & $75.8(8.6)$ \\
\hline Blood glucose (mg/dL), mean (SD) & $105.1(43.7)$ \\
\hline History of cardiovascular disease, $\mathrm{n}(\%)^{1}$ & $25(19.84)$ \\
\hline Duration of caregiving (years), mean (SD) & $4.33(3.38)$ \\
\hline Hypertension, n (\%) & $99(80.50)$ \\
\hline Dyslipidemia, n (\%) & $74(61.70)$ \\
\hline Type 2 diabetes, $\mathrm{n}(\%)$ & $23(19.17)$ \\
\hline \multicolumn{2}{|l|}{ Health Behaviors } \\
\hline Ever smoker, n (\%) & $58(46.03)$ \\
\hline Alcohol consumption (drinks/week), mean (SD) & $1.40(1.46)$ \\
\hline Meets CDC physical activity recommendation, $\mathrm{n}(\%)^{2}$ & $42(33.30)$ \\
\hline \multicolumn{2}{|l|}{ Psychological Variables } \\
\hline Role Overload score, mean (SD) & $5.18(3.15)$ \\
\hline CESD-10 score, mean (SD) & $8.78(5.81)$ \\
\hline \multicolumn{2}{|l|}{ Medication Use } \\
\hline Current use of antidepressants, n (\%) & $32(25.40)$ \\
\hline Current use of cholesterol-lowering medication, $\mathrm{n}(\%)$ & $57(45.20)$ \\
\hline Current use of high blood pressure medication, $\mathrm{n}(\%)$ & $76(60.30)$ \\
\hline Current use of diabetes medication, $n(\%)$ & $15(11.90)$ \\
\hline \multicolumn{2}{|l|}{ Objective Sleep Variables } \\
\hline Nighttime sleep duration (hours), mean (SD) & $7.32(1.12)$ \\
\hline Daytime sleep duration (hours), mean (SD) & $0.79(0.67)$ \\
\hline Nighttime WASO (hours), mean (SD) & $1.04(0.45)$ \\
\hline \multicolumn{2}{|l|}{ Subclinical Marker of Atherosclerosis } \\
\hline Mean common CIMT in millimeters, mean (SD) & $0.72(0.11)$ \\
\hline
\end{tabular}

WASO, wake after sleep Onset.

${ }^{1}$ Includes heart attack, heart failure, angina, heart disease, and stroke or transient ischemic attack.

${ }^{2}$ Engages in $\geq 30$ minutes of moderate intensity physical activity on 5 or more days of the week.

The second multivariable model included the covariates from Model 1 plus education, BMI, physical activity, smoking, hypertension, diabetes, dyslipidemia, and history of CVD. In the third model, covariates from Model 2 were included plus role overload and depression (CESD-10) scores. An alpha level of $\mathrm{p} \leq 0.05$ (2-tailed) was used to indicate statistical significance, and all statistical analyses were conducted using SPSS version 19.0.

\section{RESULTS}

\section{Participant Characteristics}

Demographic and health characteristics for the sample of spousal caregivers of a person with Alzheimer's disease are
Table 2. Sleep Parameters as Predictors of Carotid IntimaMedia Thickness in a Sample of Elderly Spousal Caregivers of a Person with Alzheimer's Disease ( $\mathrm{N}=126)$, Adjusting for Covariates

\begin{tabular}{|c|c|c|}
\hline \multirow{3}{*}{ Nighttime sleep duration (hours) } & \multicolumn{2}{|c|}{$\begin{array}{c}\text { Mean Common CIMT } \\
\text { B (95\% CI) }\end{array}$} \\
\cline { 2 - 3 } & Model 1 & $-0.01(-0.03,0.01)$ \\
\cline { 2 - 3 } & Model 2 & $-0.01(-0.03,0.01)$ \\
\cline { 2 - 3 } & Model 3 & $-0.01(-0.03,0.01)$ \\
\hline \multirow{3}{*}{ Daytime sleep duration (hours) } & Model 1 & $-0.03(-0.06,0.00)^{*}$ \\
\cline { 2 - 3 } & Model 2 & $-0.03(-0.06,-0.001)^{*}$ \\
\cline { 2 - 3 } & Model 3 & $-0.04(-0.07,-0.01)^{*}$ \\
\hline \multirow{3}{*}{ Nighttime WASO (hours) } & Model 1 & $0.05(0.01,0.09)^{*}$ \\
\cline { 2 - 3 } & Model 2 & $0.04(0.003,0.08)^{*}$ \\
\cline { 2 - 3 } & Model 3 & $0.04(0.001,0.08)^{*}$ \\
\hline
\end{tabular}

Model 1 - Adjusted for age and gender.

Model 2 - Adjusted for variables in Model 1 + education, BMI, physical activity, smoking, hypertension, dyslipidemia, diabetes, and history of CVD.

Model 3 - Adjusted for variables in Model 2 + Role Overload and depression (CESD10) scores.

$* \mathrm{p} \leq 0.05$

WASO, wake after sleep onset.

presented in Table 1. Caregivers were a mean age of 74 years, primarily women $(71 \%)$, Caucasian $(92 \%)$, slightly overweight, and had been providing care for an average of 4.3 years. The majority of caregivers had hypertension $(81 \%)$ and dyslipidemia (62\%), while $19 \%$ had Type 2 diabetes, and $20 \%$ had a history of CVD. On average, caregivers obtained 7 hours and 20 minutes of sleep and 1 hour of WASO at night, and 48 minutes of sleep during the day.

\section{Continuous Sleep Variables and Carotid Intima-Media Thickness}

In unadjusted analyses, WASO was significantly positively correlated with common CIMT $(p=0.01)$. As shown in Table 2 , in all 3 adjusted models WASO remained positively associated with CIMT (Model $1: \mathrm{B}=0.05, \mathrm{p}=0.02$; Model 2: $\mathrm{B}=0.04, \mathrm{p}=0.04$; Model 3: $\mathrm{B}=0.04, \mathrm{p}=0.05$ ). Similarly, daytime sleep duration was significantly and inversely associated with CIMT in all 3 adjusted models (Model 1: $\mathrm{B}=-0.03, \mathrm{p}=0.05$; Model 2: $\mathrm{B}=-0.03, \mathrm{p}=0.04$; Model 3: $\mathrm{B}=-0.04, \mathrm{p}=0.02$ ). Specifically, when adjusting for covariates related to sleep and atherosclerosis, for every additional hour of sleep obtained during the day, CIMT decreased by $0.04 \mathrm{~mm}$, and for every additional hour of WASO, CIMT increased by $0.04 \mathrm{~mm}$. With regard to covariates, greater CIMT was significantly correlated with increasing age, diabetes and hypertension ( $\mathrm{p}<0.05$ for all).

\section{Combined Sleep Parameters and Carotid Intima-Media Thickness}

Associations between the combined sleep parameters and CIMT were examined. As shown in Fig. (1), when adjusting for covariates included in Models 2 and 3, caregivers who obtained $<30$ minutes of daytime sleep and $\geq 1$ hour of WASO throughout the night had significantly greater common CIMT compared to caregivers who obtained $<30$ minutes of daytime sleep and $<1$ hour of WASO (reference group) ( $\mathrm{B}=0.07$ and $\mathrm{B}=0.06$, respectively; $\mathrm{p}<0.05$ for both). 


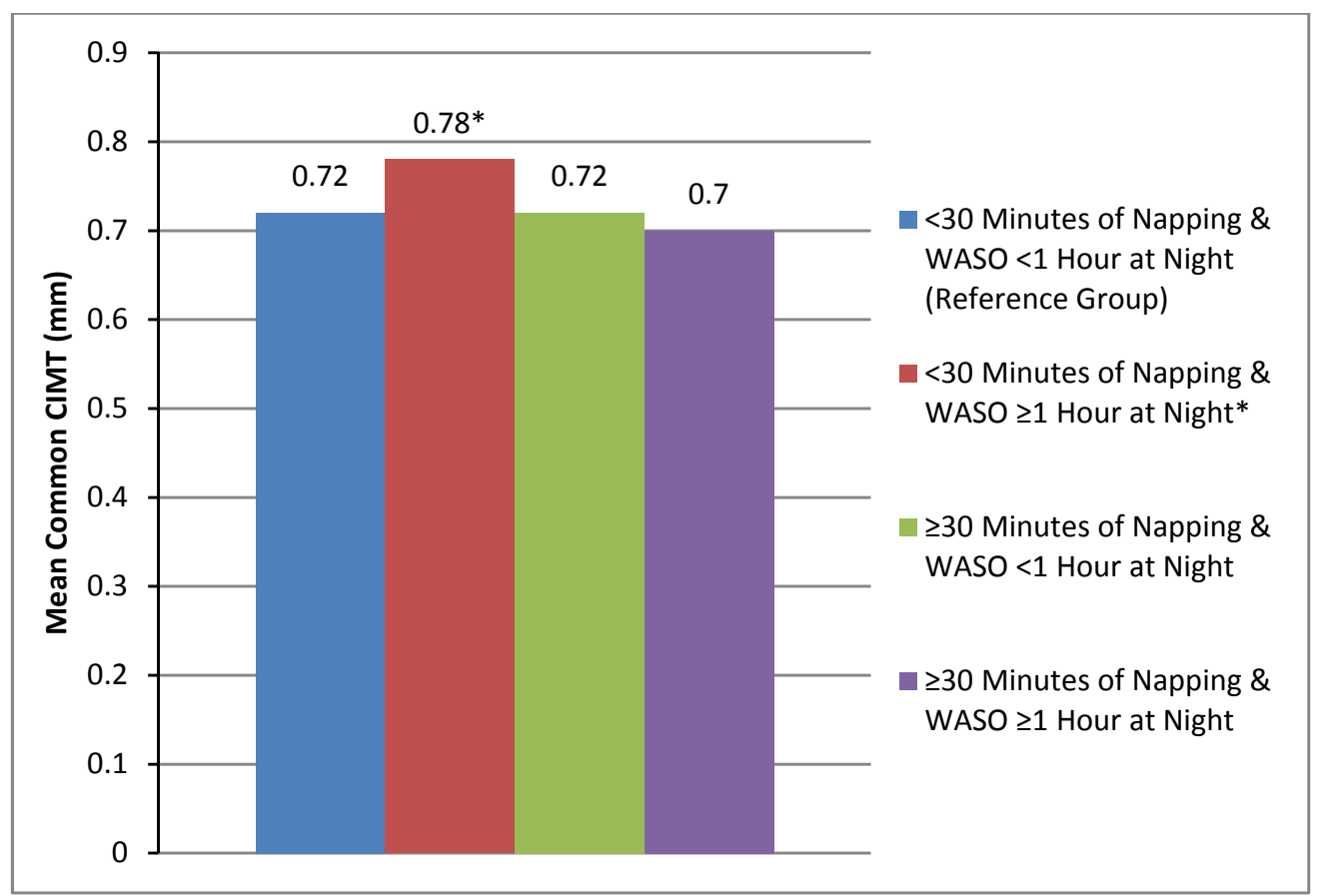

Fig. (1). Associations of combined daytime sleep duration and nighttime WASO groups with mean common carotid intima-media thickness in a sample of elderly spousal caregivers of a person with Alzheimer's disease ( $N=126)$, adjusted for covariates.

*Adjusting for covariates in Model 3, caregivers who obtained $<30$ minutes of daytime sleep and $\geq 1$ hour of WASO had significantly greater $(\mathrm{p}=0.04)$ mean common CIMT compared to the reference group.

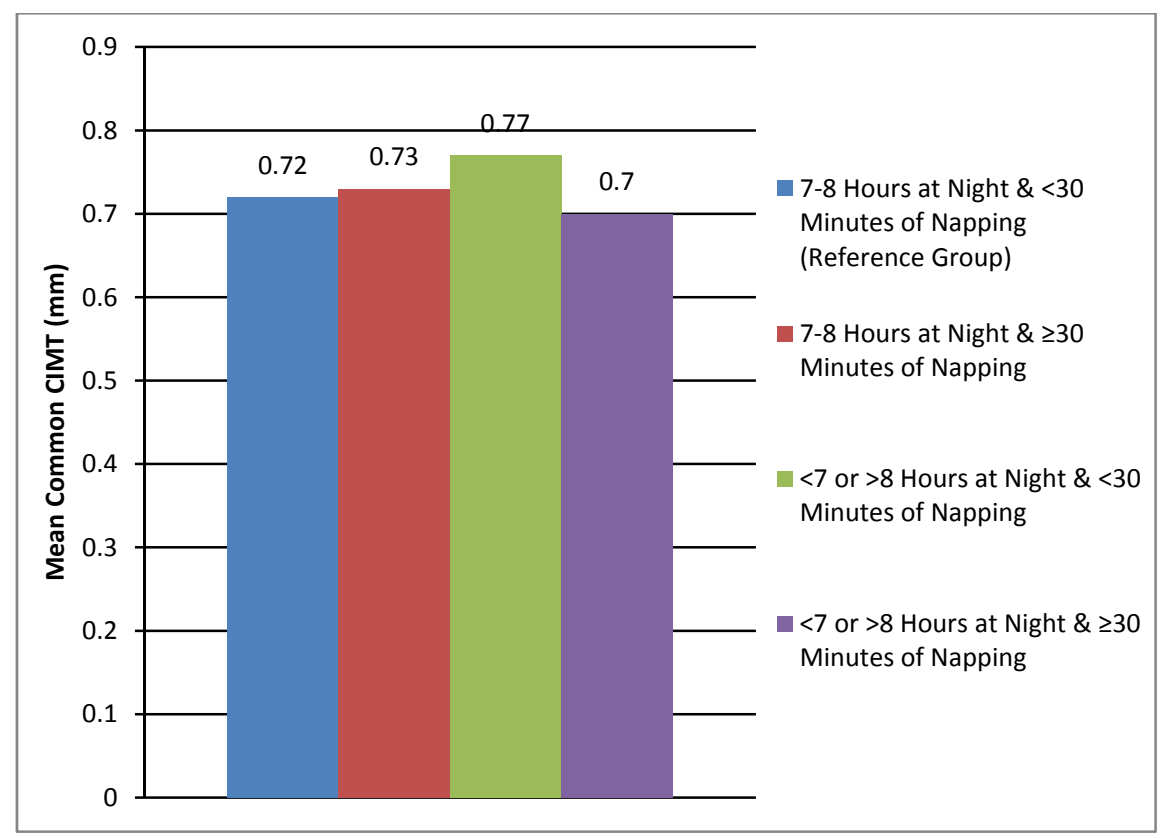

Fig. (2). Associations of combined nighttime and daytime sleep duration groups with common carotid intima-media thickness in a sample of elderly spousal caregivers of a person with Alzheimer's disease $(\mathrm{N}=126)$, adjusted for covariates.

Conversely, as shown in Figs. (2 and 3), common CIMT did not significantly differ by combined nighttime and daytime sleep duration groups, or by combined nighttime sleep duration and WASO groups, respectively.

\section{DISCUSSION}

Primary results from this analysis on elderly communitydwelling spousal caregivers of a person with Alzheimer's disease indicate that longer time spent napping was associated with significantly reduced CIMT, while longer WASO was associated with significantly increased CIMT. After adjusting for covariates related to sleep and atherosclerosis, for every additional hour spent napping during the day, CIMT decreased by $0.04 \mathrm{~mm}$, while for every additional hour of WASO, CIMT increased by $0.04 \mathrm{~mm}$. These numbers are relevant, as a reduction in CIMT by $0.1 \mathrm{~mm}$ among patients with a CIMT equal to $1.2 \mathrm{~mm}$, who are generally considered to have carotid artery atherosclerosis, would classify them as free of atherosclerosis in the carotid 


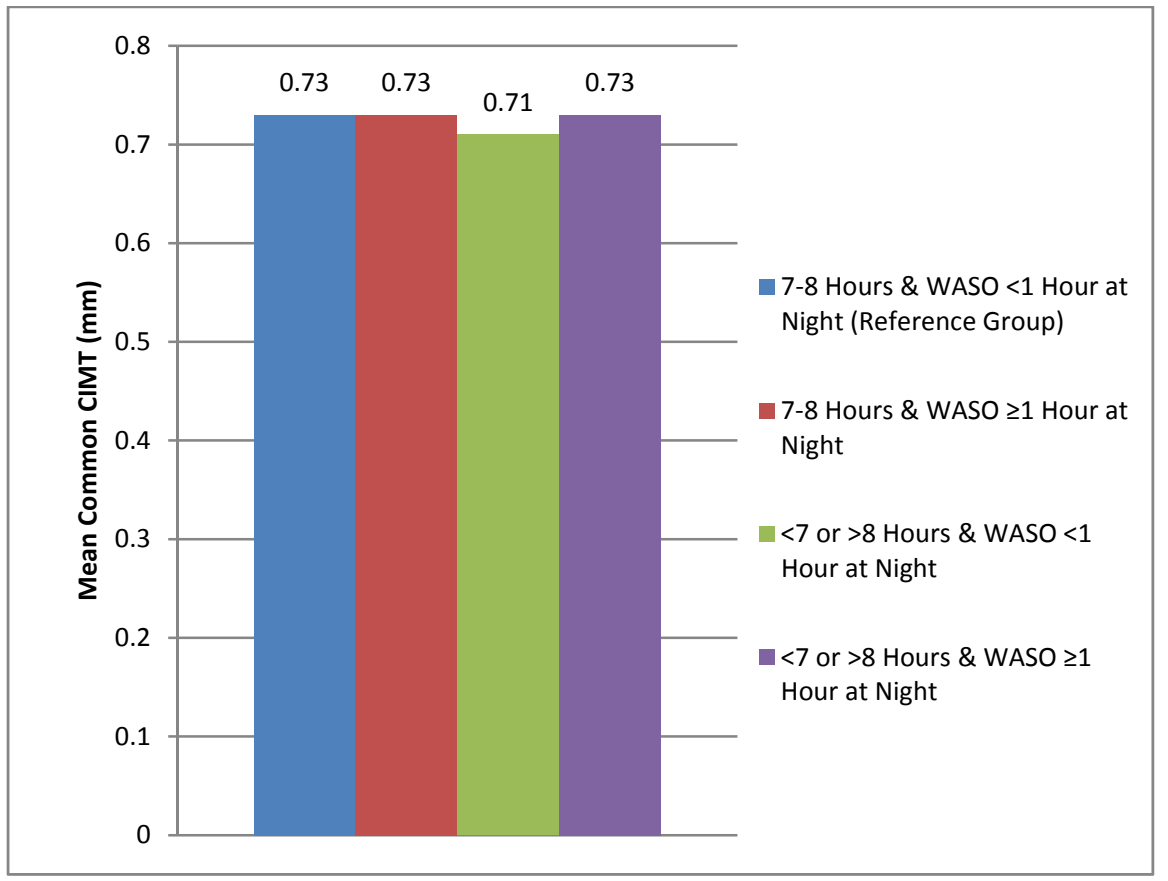

Fig. (3). Associations of combined nighttime sleep duration and nighttime WASO groups with common carotid intima-media thickness in a sample of elderly spousal caregivers of a person with Alzheimer's disease $(\mathrm{N}=126)$, adjusted for covariates.

arteries [14,17]._ENREF_32 Furthermore, a meta-analysis on data from 37,187 participants indicated that a CIMT difference of only $0.1 \mathrm{~mm}$ increased future risk of myocardial infarction and stroke by $10-15 \%$ and $15-18 \%$, respectively [14].

Our results also indicated that caregivers who napped $<30$ minutes during the day and had $\geq 1$ hour of WASO throughout the night had significantly greater CIMT compared to caregivers who napped $<30$ minutes and had $<1$ hour of WASO. This relationship appeared to be driven primarily by WASO. Therefore, our secondary analyses with combined sleep groups support findings from the primary analyses that suggest an association between WASO and CIMT.

To our knowledge, there are no studies that have investigated the relationship between objectively measured nighttime sleep disturbance and carotid artery atherosclerosis. However, our finding that WASO was significantly positively associated with CIMT is supported by previous studies reporting that disturbed sleep increases risk of cardiovascular morbidity [7, 14, 24]. For example, data from the Whitehall II Study, a large $(n=10,308)$ longitudinal cohort study on men and women, indicated that self-reported sleep disturbance significantly increased risk of coronary heart disease 15 years later [7]._ENREF_12

Our results that suggest an association between longer naps and reduced CIMT are intriguing in the face of current literature that indicates associations between longer naps and increased BMI, risk of type 2 diabetes, and mortality [25, 26]. However, since blood pressure, which predicts CIMT, acutely declines during daytime sleep, perhaps less nap time during the day leads to raised blood pressure, in turn increasing CIMT [17]. In other words, in a population of caregivers exposed to chronic stress, longer naps could provide for greater periods of reduced blood pressure, which may be protective against thickening of the carotid intimamedia [27]. It can be hypothesized that frequent periods of this acute reduction in blood pressure during a nap may be one feature explaining the reduced CIMT that was found in these elderly spousal caregivers of a person with Alzheimer's disease.

Additionally, our findings that nap duration was inversely associated with CIMT are supported by data from a large $(n=23,681)$ population-based cohort study by Naska et al., which indicated an inverse relationship between selfreported nap times and risk of coronary mortality when controlling for confounders [25]. Individuals in the Naska et al. study who napped routinely had a $37 \%$ reduced risk of coronary mortality compared to those who did not nap [25]. However, contradicting our results, analyses from a large $(n=4,797)$ study on adults over the age of 45 years indicated that longer self-reported daytime naps were associated with worse measures of subclinical atherosclerosis, but coronary artery calcium rather than CIMT was used as the marker for subclinical atherosclerosis [28].

Sleep-related atherosclerotic progression may be a potential avenue through which caregiving could predispose increased risk for CVD. Our results support findings from prior analyses that indicated associations of sleep duration and disturbed sleep with increased risk of CVD by specifically unearthing an inverse correlation between napping and CIMT, and a positive association between WASO and CIMT in elderly spousal caregivers of a person with Alzheimer's disease [7-9]. However, since the mechanisms that may mediate the presumed relationships of sleep quantity and disturbed sleep with subclinical atherosclerosis remain to be established, further research on associations between objectively measured sleep and subclinical atherosclerosis in older participants is warranted.

\section{LIMITATIONS}

Due to the cross-sectional design of the study, inferences regarding causality or directionality of associations between the sleep parameters and CIMT cannot be established. Since 
data on obstructive sleep apnea and snoring were unavailable, we were not able to adjust for this syndrome or distinguish between sleep loss due to spousal or environmental disturbances, or other pathological conditions that might disrupt sleep [29]. The caregivers in our sample had less disturbed sleep than has been observed in previous studies, they were not considered sleep deprived, and there was limited variance in the sleep variables [30]. Therefore, if the caregivers had in fact had inadequate or disturbed sleep, there may have been greater opportunity for the insufficient sleep to contribute to subclinical atherosclerotic progression, potentially resulting in stronger associations between the sleep and CIMT measures.

\section{CONCLUSIONS}

The current findings suggest that more disturbed nighttime sleep and shorter daytime naps were associated with increased CIMT in this sample of elderly spousal caregivers of a person with Alzheimer's disease. These findings provide additional information from which future prospective studies and interventions could examine whether sleep effects atherosclerotic progression over time in caregivers. Limited physical activity, poor diet, smoking, excessive alcohol consumption, and genetics certainly play an important role in the progression of atherosclerosis; but insufficient sleep, which is increasingly recognized as a modifiable risk factor and major public health concern, could also contribute $[18,31]$. A more complete theoretical model of the etiology of carotid artery IMT progression that includes sleep ought to be considered in future studies. Understanding the physiologic mechanisms that connect sleep quantity and quality to subclinical atherosclerosis has the potential to aid health care practitioners, who are ideally situated to identify and address strategies to treat sleep disturbances in caregivers, in the prevention and management of prevalent and escalating problems associated with cardiovascular risk factors and CVD.

\section{CONFLICT OF INTEREST}

The authors confirm that this article content has no conflicts of interest.

\section{ACKNOWLEDGEMENT}

Declared none.

\section{REFERENCES}

[1] Rowe MA, McCrae CS, Campbell JM, Benito AP, Cheng J. Sleep pattern differences between older adult dementia caregivers and older adult noncaregivers using objective and subjective measures. J Clin Sleep Med 2008; 4(4): 362-9.

[2] Aschbacher K, Mills PJ, von Känel R, et al. Effects of depressive and anxious symptoms on norepinephrine and platelet P-selectin responses to acute psychological stress among elderly caregivers. Brain Behav Immun 2008; 22(4): 493-502.

[3] Mausbach BT, Ancoli-Israel S, von Känel R, et al. Sleep disturbance, norepinephrine, and D-dimer are all related in elderly caregivers of people with Alzheimer disease. Sleep 2006; 29(10): 1347-52.

[4] Lusardi P, Mugellini A, Preti P, Zoppi A, Derosa G, Fogari R. Effects of a restricted sleep regimen on ambulatory blood pressure monitoring in normotensive subjects. Am J Hypertens 1996; 9(5): 503-5.
[5] National Alliance for Caregiving, American Association of Retired Persons. Caregiving in the U.S.: Executive summary. Bethesda, MD: National Alliance for Caregiving 2005.

[6] Wolk R, Gami AS, Garcia-Touchard A, Somers VK. Sleep and cardiovascular disease. Curr Probl Cardiol 2005; 30(12): 625-62.

[7] Chandola T, Ferrie JE, Perski A, Akbaraly T, Marmot MG. The effect of short sleep duration on coronary heart disease risk is greatest among those with sleep disturbance: a prospective study from the Whitehall II cohort. Sleep 2010; 33(6): 739-44.

[8] Werle MH, Moriguchi E, Fuchs SC, Bruscato NM, de Carli W, Fuchs FD. Risk factors for cardiovascular disease in the very elderly: results of a cohort study in a city in southern Brazil. Eur J Cardiovasc Prev Rehabil 201;18(3): 369-77.

[9] Cappuccio FP, D’Elia L, Strazzullo P, Miller MA. Sleep duration and all-cause mortality: a systematic review and meta-analysis of prospective studies. Sleep 2010; 33(5): 585-92.

[10] Choi KM, Lee JS, Park HS, Baik SH, Choi DS, Kim SM. Relationship between sleep duration and the metabolic syndrome: Korean National Health and Nutrition Survey 2001. Int J Obes (Lond) 2008; 32(7): 1091-7.

[11] Kannel WB. Coronary heart disease risk factors in the elderly. Am J Geriatr Cardiol 2002;11(2): 101-7.

[12] Finn AV, Kolodgie FD, Virmani R. Correlation between carotid intimal/medial thickness and atherosclerosis: a point of view from pathology. Arterioscler Thromb Vasc Biol 2010; 30(2): 177-81.

[13] O'Leary DH, Polak JF, Kronmal RA, Manolio TA, Burke GL, Wolfson SK Jr. Carotid-artery intima and media thickness as a risk factor for myocardial infarction and stroke in older adults. Cardiovascular Health Study Collaborative Research Group. N Engl J Med 1999; 340(1): 14-22.

[14] Lorenz MW, Markus HS, Bots ML, Rosvall M, Sitzer M. Prediction of clinical cardiovascular events with carotid intimamedia thickness: a systematic review and meta-analysis. Circulation 2007; 115(4): 459-67.

[15] Wolff B, Völzke H, Schwahn C, Robinson D, Kessler C, John U. Relation of self-reported sleep duration with carotid intima-media thickness in a general population sample. Atherosclersis 2008; 196(2): 727-32.

[16] Ramos-Sepulveda A, Wohlgemuth W, Gardener H, et al. Snoring and insomnia are not associated with subclinical atherosclerosis in the Northern Manhattan Study. Int J Stroke 2010; 5(4): 264-8.

[17] Abe T, Aoki T, Yata S, Okada M. Sleep duration is significantly associated with carotid artery atherosclerosis incidence in a Japanese population. Atherosclerosis 2011; 217(2): 509-13.

[18] von Känel R, Ancoli-Israel S, Dimsdale JE, et al. Sleep and biomarkers of atherosclerosis in elderly Alzheimer caregivers and controls. Gerontology 2010; 56(1): 41-50.

[19] Kring SII, Brummett BH, Barefoot J, et al. Impact of psychological stress on the associations between apolipoprotein E variants and metabolic traits: findings in an American sample of caregivers and controls. Psychosom Med 2010; 72(5): 427-33.

[20] Diagnosis and classification of diabetes mellitus. Diabetes Care 2009; 32(Suppl 1): S62-7.

[21] Hamilton M. A rating scale for depression. J Neurol Neurosurg Psychiatr 1960; 23: 56-62.

[22] Ancoli-Israel S, Cole R, Alessi C, Chambers M, Moorcroft W, Pollak CP. The role of actigraphy in the study of sleep and circadian rhythms. Sleep 2003; 26(3): 342-92.

[23] Stone KL, Ewing SK, Ancoli-Israel S, et al. Self-reported sleep and nap habits and risk of mortality in a large cohort of older women. J Am Geriatr Soc 2009; 57(4): 604-11.

[24] Meisinger C, Heier M, Löwel H, Schneider A, Döring A. Sleep duration and sleep complaints and risk of myocardial infarction in middle-aged men and women from the general population: the MONICA/KORA Augsburg cohort study. Sleep 2007; 30(9): 1121-7. [25] Naska A, Oikonomou E, Trichopoulou A, Psaltopoulou T, Trichopoulos D. Siesta in healthy adults and coronary mortality in the general population. Arch Intern Med 2007; 167(3): 296-301.

[26] Bursztyn M, Stessman J. The siesta and mortality: twelve years of prospective observations in 70-year-olds. Sleep 2005; 28(3): 345-7. Dechering DG, Wizner B, Adiyaman A, et al. Sphygmomanometric and ambulatory blood pressures as forerunners of carotid and femoral intima-media thickness. J Hypertens 2009; 27(4): 81321. 
[28] Stang A, Dragano N, Poole C, et al. Daily siesta, cardiovascular risk factors, and measures of subclinical atherosclerosis: results of the Heinz Nixdorf Recall Study. Sleep 2007; 30(9): 1111-9.

[29] Lee SA, Amis TC, Byth K, et al. Heavy snoring as a cause of carotid artery atherosclerosis. Sleep 2008; 31(9): 1207-13.

[30] Von Känel R, Dimsdale JE, Ancoli-Israel S, et al. Poor sleep is associated with higher plasma proinflammatory cytokine interleukin-6 and procoagulant marker fibrin D-dimer in older caregivers of people with SAlzheimer's disease. J Am Geriatr Soc 2006; 54(3): 431-7.

[31] Vgontzas AN, Liao D, Bixler EO, Chrousos GP, Vela-Bueno A. Insomnia with objective short sleep duration is associated with a high risk for hypertension. Sleep 2009; 32(4): 491-7.

Received: June 27, 2012

(C) Schwartz et al.; Licensee Bentham Open.

This is an open access article licensed under the terms of the Creative Commons Attribution Non-Commercial License (http://creativecommons.org/licenses/by$\mathrm{nc} / 3.0 /$ ), which permits unrestricted, non-commercial use, distribution and reproduction in any medium, provided the work is properly cited. 